\title{
Bem-estar animal de cães durante as intervenções assistidas por animais
}

\author{
Animal welfare of dogs during animal assisted interventions
}

Bienestar animal de perros durante las intervenciones asistidas por animals

Recebido: 27/10/2020 | Revisado: 05/11/2020 | Aceito: 13/01/2021 | Publicado: 15/01/2021

Fernanda Dagmar Martins Krug

ORCID: https://orcid.org/0000-0001-9123-9399

Universidade Federal de Pelotas, Brasil

E-mail:fernandadmkrug@gmail.com

Sabrina de Oliveira Capella

ORCID: https://orcid.org/0000-0001-8252-2096

Universidade Federal de Pelotas, Brasil

E-mail: capellas.oliveira@gmail.com

Clederson Idenio Schmitt

ORCID: https://orcid.org/0000-0001-7948-9627 Universidade Federal de Pelotas, Brasil E-mail: schmittproducoes@gmail.com

Beatriz Liboni Alcalá Freguglia

ORCID: https://orcid.org/0000-0002-2644-4254 Universidade Federal de Pelotas, Brasil

E-mail: bia.alcala@gmail.com

Edgar Cleiton da Silva

ORCID: https://orcid.org/0000-0001-5329-5277 Universidade Federal de Pelotas, Brasil

E-mail: edgar.cleiton@gmail.com

Camila Moura de Lima

ORCID: https://orcid.org/0000-0001-9306-705X

Universidade Federal de Pelotas, Brasil

E-mail: camila.moura.lima@hotmail.com

Márcia de Oliveira Nobre

ORCID: https://orcid.org/0000-0003-3284-9167 Universidade Federal de Pelotas, Brasil

E-mail: marciaonobre@gmail.com

\section{Resumo}

Este estudo demonstra os efeitos fisiológicos e comportamentais dos cães que atuam como coterapeutas em Intervenções Assistidas por Animais (IAAs). A maioria das pesquisas se concentra nos assistidos durante as IAAs, não levando em conta os coterapeutas. Assim, buscou-se avaliar os efeitos das IAAs sobre os parâmetros fisiológicos e comportamentais dos cães coterapeutas. Foram realizadas avaliações de pressão arterial sistólica, pressão arterial diastólica, temperatura, frequência cardíaca, frequência respiratória e comportamento de cinco cães coterapeutas do projeto Pet Terapia da Universidade Federal de Pelotas em três locais diferentes. As avaliações comportamentais foram registradas por meio de filmagens durante visitas às instituições e posteriormente registradas em etograma. Os resultados obtidos durante a avaliação dos sinais vitais mostraram que não houve alterações ou quando houve relação com a redução da pressão arterial dos cães, os cães não apresentaram comportamentos relacionados ao estresse, tais como: vocalização, comportamento passivo, ativo/repetitivo. Conclui-se que intervenções assistidas em animais, desenvolvidas com cães treinados e adaptados aos moradores e ao público assistido durante o período de 40 a $60 \mathrm{~min}$, com equipe treinada não causam alterações nos sinais vitais e no comportamento dos cães coterapeutas, indicando estresse, garantindo assim saúde e bem-estar animal.

Palavras-chave: Comportamento animal; Cães coterapeutas; Medicina veterinária; Pet terapia; Etograma; Sinais vitais.

\begin{abstract}
This study demonstrates the physiological and behavioral effects of dogs that act as co-therapists in Animal Assisted Interventions (AAT). Most research focuses on those assisted during AAT, disregarding co-therapists. Thus, we sought to evaluate the effects of AAT on the physiological and behavioral parameters of the dog co-therapists. Assessments of systolic blood pressure, diastolic blood pressure, temperature, heart rate, respiratory rate and behavior of five co-therapist dogs from the Pet Terapia project at the Federal University of Pelotas were carried out in three different locations. Behavioral assessments were recorded by filming during visits to the institutions and later recorded on an ethogram. The results obtained during the evaluation of vital signs showed that there were no changes or when there was a relationship with the reduction of blood pressure in dogs, the dogs did not show behaviors related to stress, such as: vocalization, passive, active / repetitive
\end{abstract}


behavior. It is concluded that assisted interventions in animals, developed with trained dogs and adapted to the residents and the assisted public during the period of 40 to $60 \mathrm{~min}$, with trained staff do not cause changes in vital signs and behavior of co-therapist dogs, indicating stress, guaranteeing stress thus animal health and welfare.

Keywords: Animal behavior; Dogs therapists, Veterinary medicine; Pet therapy; Ethogram; Vital signs.

\begin{abstract}
Resumen
Este estudio demuestra los efectos fisiológicos y conductuales de los perros que actúan como coterapeutas en las intervenciones asistidas por animales (AII). La mayor parte de la investigación se centra en las personas asistidas durante las AII, sin tener en cuenta a los coterapeutas. Por lo tanto, buscamos evaluar los efectos de la AII sobre los parámetros fisiológicos y de comportamiento de los coterapeutas caninos. Se llevaron a cabo evaluaciones de presión arterial sistólica, presión arterial diastólica, temperatura, frecuencia cardíaca, frecuencia respiratoria y comportamiento de cinco perros coterapeutas del proyecto Pet Terapia de la Universidad Federal de Pelotas en tres ubicaciones diferentes. Las evaluaciones de comportamiento se registraron filmando durante las visitas a las instituciones y luego se registraron en una etograma. Los resultados obtenidos durante la evaluación de signos vitales mostraron que no hubo cambios o cuando hubo una relación con la reducción de la presión arterial en los perros, los perros no mostraron conductas relacionadas con el estrés, tales como: vocalización, comportamiento pasivo, activo / repetitivo. Se concluye que las intervenciones asistidas en animales, desarrolladas con perros adiestrados y adaptadas a los residentes y público asistido durante el período de 40 a 60 min, con personal adiestrado, no provocan cambios en los signos vitales y comportamiento de los perros coterapeutas, indicando estrés, garantizando estrés. de ahí la salud y el bienestar de los animales.

Palabras clave: Comportamiento animal; Terapeutas de perros, Medicina veterinaria; Terapia de mascotas; Etograma; Signos vitales.
\end{abstract}

\title{
1. Introdução
}

As primeiras ideias do uso de animais para fins terapêuticos começaram a surgir na Europa, com filantropo William Tuke, que começou a usar animais domésticos para auxiliar seus pacientes psiquiátricos em suas tarefas diárias (Dotti, 2014). No Brasil, essa prática, denominada Intervenções Assistidas por Animais (IAAs), teve início em 1950 com a psiquiatra médica Nise da Silveira (Chelini \& Otta, 2016). As IAAs estão bastante consolidadas em diversos países, como Espanha, Estados Unidos da América, entre outros (Serpell, et al., 2010). Essa prática é conhecida como atividade assistida por animais, o qual refere-se a qualquer uso de animais treinados para o benefício terapêutico, motivacional ou educacional dos pacientes (Kruger, et al., 2010).

Programas de atividade assistida por animais (e terapia) têm sido associados a efeitos positivos aos humanos em muitos estudos (Clarck, et al., 2019; BERT, et al., 2016), e no Brasil, ela vem atraindo interesse por profissionais da área da saúde e educação, e vem sendo feitos estudos para comprovar os efeitos benéficos da IAAs na saúde física, mental e social dos indivíduos atendidos (Nobre, et al., 2018). O campo da terapia assistida por animais está crescendo exponencialmente sob a falta de evidências científicas de riscos potenciais para os animais envolvidos (Clarck, et al., 2019). E quando analisamos especificamente o Brasil, são poucos relatos sobre a saúde e o bem-estar dos animais envolvidos, uma vez que o estresse pode ser gerado durante a interação entre o público-alvo e os cães participantes (Vasconselos, et al., 2016; Yamamoto, et al., 2012). Além disso a maioria das pesquisas no Brasil são relacionados aos assistidos.

Mais ainda que é de extrema importância garantir não só a saúde e o bem-estar dos pacientes e alunos atendidos, mas também dos cães coterapeutas envolvidos (Nobre, et al., 2018; Rosa, et al., 2015). Para isso, é necessário avaliar indicadores de bemestar animal, como avaliação de sinais vitais e comportamentais (através da linguagem corporal). Além do acompanhamento de um veterinário responsável pela periodicidade da avaliação clínica e comportamental, exames complementares, vacinação e higiene (Lima, et al., 2018). Mais ainda quando esses cães realizam o trabalho de pet terapia em ambientes complexos, como um ambiente hospitalar, ambiente escolar e associado a isso estão os diferentes públicos, por essas questões devemos compreender o bem-estar de cães de terapia (Clarck, et al., 2019). Diante disso, o presente estudo tem como objetivo avaliar o bem-estar animal dos cães coterapeutas do Projeto de Terapia da Universidade Federal de Pelotas-RS durante as Intervenções Assistidas por Animais (IAAs). 


\section{Metodologia}

O presente estudo foi aprovado pelo Comitê de Ética e Experimentação Animal da UFPel (5612) e cinco cães sem raça definida, que ficam no canil do projeto junto a universidade, foram utilizados como coterapeutas do projeto Pet Terapia da Faculdade de Medicina Veterinária da Universidade Federal de Pelotas. Para o desenvolvimento da AHAS, os cães passavam por um controle sanitário e de higiene, seguindo as recomendações/protocolos de higiene de cada instituição. Além disso, eles passavam por capacitação para a realização das atividades propostas nas instituições, através de treinamentos semanais, sempre com acompanhamento veterinário durante todo o processo de capacitação e nas intervenções. As intervenções assistidas por animais duraram de 40 a 60 min e ocorreram semanalmente em dois hospitais (HO1 e HO2) e uma escola municipal (MS), ambas instituições públicas, de junho a novembro de 2017 na cidade de Pelotas - RS, Brasil.

Em relação aos locais onde ocorriam as atividades dentro das instituições, no HO1 as visitas ocorreram na sala de espera disponibilizada pela instituição. No $\mathrm{HO} 2$, as visitas ocorreram em uma sala destinada à oficina terapêutica, espaço esse contava com mesas, bancos e um jardim ao ar-livre. Na MS as visitas ocorreram em uma sala de recursos pedagógicos, com mesas, cadeiras e materiais pedagógicos.

O público atendido pelas IAAs, foram bem diversificados, sendo no HO1 o público atendido foram crianças de seis meses a 12 anos de idade, tendo em média cada visita o atendido de oito crianças com diferentes idades. As quais permaneciam sentadas nas cadeiras durante as IAAs, e na grande maioria tinham acesso venoso com cateter, outras com talas em algum membro e também crianças com cadeiras de rodas. No $\mathrm{HO} 2$, eram atendidos um número variável de pacientes adultos com diversos transtornos mentais, porquê decorria das internações de rotinas do hospital, tendo a média de quatro a seis pacientes por sessão. Na MS, o público eram três de crianças de idades diferentes, ambas com diferentes graus do transtorno do espectro autista (TEA).

As atividades realizadas nas instituições, eram propostas com a equipe multidisciplinar das instituições juntamente com o projeto Pet Terapia da UFPel. No H01 propostas aos pacientes, iniciava com a abordagem, toque nos cães e foram estimulados por meio de atividades de cuidado, escovação de pelos e brincadeiras, como simulação de procedimentos médicos, como ouvir o coração, aplicar medicamentos e colocar ataduras em cães coterapeutas, jogos de memória e quebra-cabeças. No H02, as atividades propostas aos pacientes eram: realização os passeios com os cães acompanhados dos integrantes do Pet Terapia da oficina até o jardim, passavam algum tempo no colo dos pacientes recebendo carinho, escovando os pelos e sendo acariciados. Posteriormente, foram realizadas as atividades lúdicas, sempre relacionadas aos cães do projeto, onde os cães permaneciam com os pacientes. No MS como as visitas ocorreram em uma sala de recursos pedagógicos, as atividades sempre eram preconizadas os objetivos a serem atingidos de cada aluno, já que possuíam diferentes graus de autismo. Dentre das atividades realizadas, eram caminhadas, pareamento de letras e números, leitura aos cães, etc... Nos três locais, sempre foi preconizado o bem-estar animal durante as atividades propostas e sempre as atividades eram avaliadas pela médica veterinária residente do projeto.

Para verificar o bem-estar dos cães coterapeutas durante as intervenções, foram realizadas avaliações de sinais vitais paramétricos, com dez visitas nas instituições mencionadas acima. Os cães foram identificados como 01,02, 03, 04 e 05. Cada um desses cães apresentava características específicas que foram utilizadas e estimuladas durante a IAAs. Todos os cães tinham um perfil calmo e receptivo, sendo todas fêmeas, de adulto a adulto maduro. $\mathrm{O}$ cão $\mathrm{n}^{\circ} 03$ era um cão mais calmo que os outros participantes e o cão 05 era de um perfil mais ativo, com energia suficiente para atividades que necessitasse mais energia, como caminhadas. Considerando as características específicas de cada um dos cães, e as atividades propostas nas instituições foram respeitado o tempo de atividade/trabalho de cada cão, assim como as horas de descanso. Como os locais onde são realizadas as atividades não dispõe de espaço adequado que proporcionasse uma adequada avaliação dos parâmetros vitais dos cães, adotou-se as 
Research, Society and Development, v. 10, n. 1, e3001019730, 2021

(CC BY 4.0) | ISSN 2525-3409 | DOI: http://dx.doi.org/10.33448/rsd-v10i1.9730

avaliações dos sinais vitais do cães em momentos diferentes: $M_{0}$ - medição no ambiente doméstico (Canil do projeto Pet Terapia), 30 minutos antes de embarcar no transporte para ir às instituições para o desenvolvimento das atividades; $\mathrm{M}_{1}$ - imediatamente após o retorno (viagem de carro) para o ambiente doméstico. Em cada um dos momentos foram verificados: temperatura corporal (TR) (por termômetro retal), frequência cardíaca (FC) (bpm - batidas por minuto), frequência respiratória (FR) (mpm - estetoscópio), pressão arterial sistólica (PAS) e pressão arterial diastólica (PAD) do membro posterior direito com o dispositivo digital Tech Line (método não invasivo). Para evitar que a avaliação da pressão ocasionasse um estresse aos cães, eles foram condicionados por dez dias, sendo realizada a para a verificação da pressão arterial, assim não provocasse algum estresse durante as avaliações posteriores.

Para avaliar o comportamento dos animais, as intervenções foram filmadas com auxílio de uma câmera GORPO ${ }^{\circledR}$ por 15 minutos cada cão, e em cada instituição. Posteriormente as imagens foram avaliadas a partir de um etograma (Figura 1) adaptado de Pereira (2015), onde os comportamentos observados foram registrados nele. Para uma melhor avaliação do comportamento, foi feita adaptações do etograma, sendo excluídas as categorias comportamentais de posição na caixa e manutenção, uma vez que esse tipo de comportamento não ocorre nas IAAs. 
Research, Society and Development, v. 10, n. 1, e3001019730, 2021

(CC BY 4.0) | ISSN 2525-3409 | DOI: http://dx.doi.org/10.33448/rsd-v10i1.9730

Figura 1. Linguagem corporal do cão através da avaliação postura, posição da cauda, orelhas, olhos e cabeça.

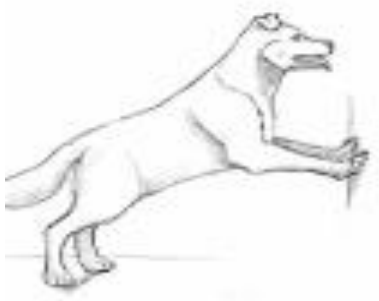

Empinado

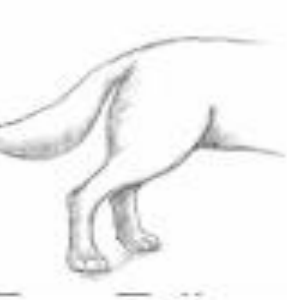

Cauda Tensa

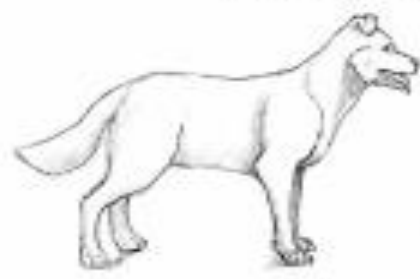

Em Pè

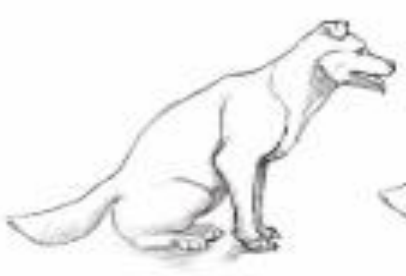

Sentado

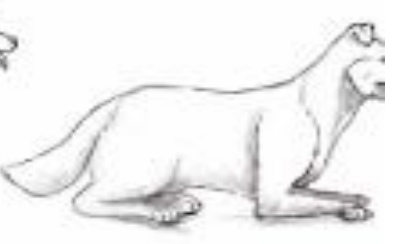

Deitado

Posicaoda Canda do Cáo

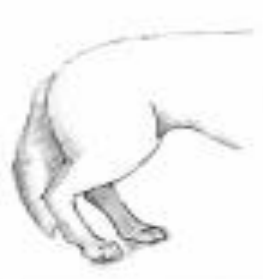

Cauda Baixat

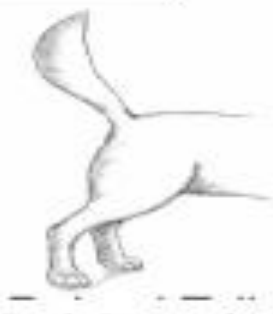

Cauda Levantada

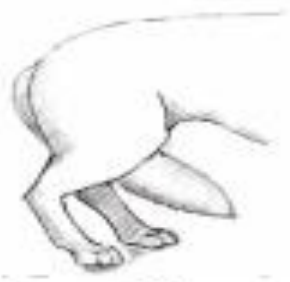

Cauda no meio das pernas

Posicao das Orelhas do Cao

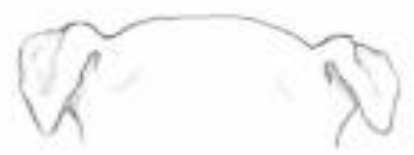

Orelhas Relaxadas

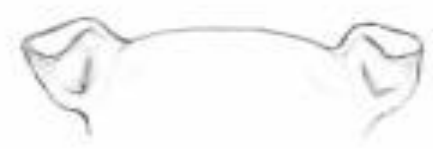

Orelhas em Posiçâo de Alerta

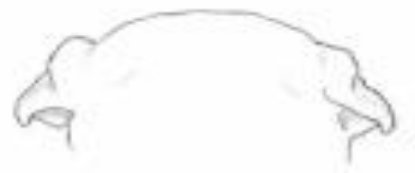

Orelhas Abaixadas

\section{Posicào dos Olhos do Cão}
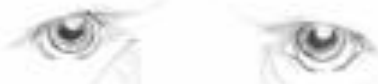

Olhar Indireto do Cåo

Olhar de Alerta do Cáo
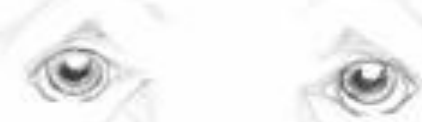

\section{Posicào da Cabeca do Cào}

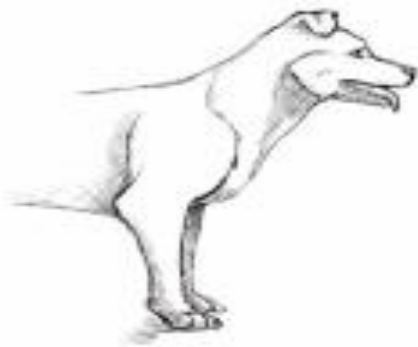

Cabeça Levantada

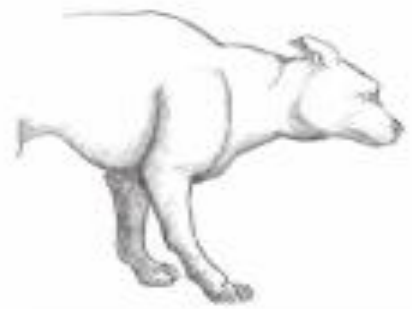

Cabeça Baixa

Fonte: Autores. 
Também foram incluídas imagens da linguagem corporal dos cães, para uma melhor compreensão dos avaliadores. Os comportamentos dos cães foram avaliados pela linguagem corporal dos cães e analisados por três avaliadores á cegas a cada cinco minutos da filmagem. O etograma foi dividido em categorias comportamentais, primeira parte através da linguagem corporal (Figura 1): como postura (pé, sentado/deitado, íngreme/colo), posição dos olhos (relaxado, alerta e indireto), posição dos ouvidos relaxados, alerta e para trás), posição da cauda (levantada, tensa, entre as pernas), posição da cabeça (levantada, baixada).

$\mathrm{Na}$ segunda parte das categorias comportamentais, vocalizações (ausentes, resmungar, urinar, rosnar), interação com humanos (ausente, brincar, lamber, pata, observar, assustar, agressivo) interação com o ambiente (interagir, brincar, rolar, empurrar, cavar o chão, cheirar), comportamentos ativos/ repetitivos (andar, andar, dormir, andar) estereotipar, andar em círculos, perseguir cauda, pular, balançar, lamber), comportamento típico de estresse (bocejo, lamber lábios, latir, afar, automutilação). As médias dos dados paramétricos foram obtidas pelo Programa R e a frequência dos comportamentos pelo programa SSPS STATISTICS.

\section{Resultados e Discussão}

Sabe-se que as atividades com os cães, nas IAAs são solicitados a realizar várias tarefas, como interagir com pessoas desconhecidas em ambientes desconhecidos (Mazzoti, et al., 2009; Riemer, et al., 2006), e há o potencial para que essas interações causem estresse para animais de terapia (Serpell, et al., 2010). E analisando os sinais vitais médios dos cinco cães coterapeutas estudados permaneceram normais quando comparados antes e depois do trabalho e no HO2 houve redução significativa nas sistólicas $(\mathrm{p}=0,0001)$ e diastólicas $(\mathrm{p}=0,0001)$. Resultados esses são semelhantes ao estudo de Melco et al. (2020), que ao utilizarem cães nas IAAs com crianças com déficit de atenção em secções de 20 min, não observaram alterações fisiológicas nos cães. Ao comparar esse estudo com o nosso, os cães realizam o dobro de atividades nas IAAs o que poderia ter ocorrido estresse, no entanto não ocorreu. Isso seja porquê a equipe que trabalha com os cães participa de todo o processo de treinamento dos cães e com isso eles conseguem identificar qualquer alteração comportamental.

Podemos enfatizar de forma geral que nas três instituições os cães coterapeutas mantiveram seus parâmetros sinais vitais dentro dos padrões esperados para as espécies. Confirmando que todos estavam sem a manifestação de estresse, pois quando o corpo está sob estresse, a liberação de cortisol do eixo hipotálamo-hipófise adrenal aumenta, podendo causar alguns resultados prejudiciais à saúde e ao bem-estar (Fries, et al., 2009; Glenk, et al., 2013). Como não ocorreu alteração nos parâmetros vitais, não ocorreu estresse, já que ele ocasiona aumento dos níveis de cortisol e com essa taquicardia (Vasconcelos, 2016).

O ligeiro aumento da temperatura retal pode ser justificado em função do exercício, já que no HO2 havia uma área externa de jardim, onde as caminhadas estavam associadas ao retorno dos cães ao seu ambiente doméstico, o que pode causar certa excitação. Resultados semelhantes foram observados por Clarck et al. (2020) que ainda salientam que muitos fatores podem influenciar a temperatura corporal do cão, como por exemplo a idade, sexo, tamanho do corpo, temperatura ambiente e esforço físico. Com isso, fica evidente que esse aumento da temperatura retal observada foi decorrente das atividades mais intensas realizadas junto com os assistidos. 
Research, Society and Development, v. 10, n. 1, e3001019730, 2021

(CC BY 4.0) | ISSN 2525-3409 | DOI: http://dx.doi.org/10.33448/rsd-v10i1.9730

Tabela 1. Média dos sinais vitais dos cães medidos antes e depois das visitas às instituições.

\begin{tabular}{lllllcccccc}
\hline \multirow{2}{*}{ Sinais } & \multicolumn{3}{c}{ HO1 } & \multicolumn{3}{c}{ HO2 } & \multicolumn{3}{c}{ MS } \\
\cline { 2 - 9 } Vitais & \multicolumn{3}{c}{ Média } & \multicolumn{3}{c}{ Média } & & Média \\
& \cline { 2 - 10 } & Antes & Depois & p-valor & Antes & Depois & p-valor & Antes & Depois & p-valor \\
\hline FR & 25,3 & 49,1 & 0,1 & 35,6 & 36 & 0,933 & 37,5 & 33,8 & 0,51 \\
FC & 98,1 & 93,1 & 0,2 & 94,03 & 98,3 & 0,421 & 99,8 & 87,8 & 0,08 \\
TR & 37,9 & 38,3 & 0,06 & 37,7 & 38,02 & 0,0001 & 38,1 & 38,1 & 0,81 \\
PAS & 132,5 & 131,8 & 0,9 & 141,2 & 125,4 & 0,001 & 147,6 & 142,2 & 0,32 \\
PAD & 93,6 & 96,2 & 0,7 & 102,9 & 87,19 & 0,001 & 98,9 & 94,06 & 0,38 \\
\hline
\end{tabular}

Abreviaturas: HO1: Hospital número 01; HO2: Hospital número 02; PAD: Pressão Sanguínea Diastólica; FC: Frequência cardíaca; MS: Escola Municipal; FR: Frequência Respiratória; TR: Temperatura retal; PAS: Pressão Arterial Sistólica. Fonte: Autores.

Quando avaliamos a pressão arterial sistólica (PAS) (Tabela 2) de cada cão em relação à população-alvo de cada instituição, obtivemos uma redução significativa das pressões dos cães 04 e 05 na instituição HO2. Nas demais instituições podemos considerar que os outros cães também apresentaram diminuição das pressões medidas antes e depois das visitas, quando relacionadas à pressão arterial fisiológica de cada animal em dias que não estão realizando atividades. Esses resultados mostraram que o trabalho dos cães na IAAs não interferiu na temperatura, frequência cardíaca, frequência respiratória e até mesmo na instituição HO2, os cães foram mais relaxados e relaxados durante e após as atividades (Tabela 1). Resultados esses obtidos, vão ao encontro dos resultados obtidos por Clarck et al. (2020), os quais enfatizam essa redução da pressão sistólica e diastólica baixa após as secções é significativo dos cães estarem em um estado mais relaxado após a secção.

Quanto ao comportamento dos cães coterapeutas no HO1, os cães 01, 02 e 03 foram mantidos durante a parte IAAs do tempo de pé e a outra metade do tempo, com posição dos olhos e ouvidos oscilando entre alerta e relaxado, bem como cauda de alerta e cabeça levantada. Os três cães interagiram com os pacientes, o cão um ainda pediu carinho aos pacientes, estendendo as patas e o cão 02 também explorou o ambiente e ficou curioso. 
Tabela 2. Pressão arterial sistólica média (PAS) de cães coterapeutas em dias sem atividades e nas instituições considerando o antes e após as visitas.

\begin{tabular}{|c|c|c|c|c|c|c|c|c|c|c|}
\hline \multirow{3}{*}{ Cão } & \multirow{3}{*}{ PSSM } & \multicolumn{4}{|c|}{ HO1 } & \multicolumn{3}{|c|}{$\mathrm{HO} 2$} & \multicolumn{2}{|c|}{ MS } \\
\hline & & \multicolumn{4}{|c|}{ Média (PAS) } & \multicolumn{3}{|c|}{ Média (PAS) } & \multicolumn{2}{|c|}{ Média (PAS) } \\
\hline & & Antes & Depois & $\mathrm{p}$-valor & Antes & Depois & p-valor & Antes & Depois & p-valor \\
\hline 01 & 160 & 143,1 & 131,5 & 0,8 & - & - & - & 150 & 140,3 & 0,5 \\
\hline 02 & 137 & 134,4 & 123,3 & 0,4 & 145,05 & 143,3 & 0,07 & - & - & - \\
\hline 03 & 114 & 116 & 129,5 & 0,3 & 117,9 & 101,2 & 0,002 & - & - & - \\
\hline 04 & 160 & - & - & - & 153 & 1128,7 & 0,02 & 150 & 145,1 & 0,3 \\
\hline 05 & 150 & - & - & - & 153 & 133,6 & 0,02 & 128 & 137,2 & 0,4 \\
\hline
\end{tabular}

Abreviaturas: HO1: Hospital número 01; HO2: Hospital número 02; MS: Escola Municipal; PAS: Pressão Arterial Sistólica; PSSM: Média da Pressão Arterial Sistólica Fisiológica dos Cães. Fonte: Autores.

No H02 a postura dos cães 02, 03, 04 e 05 variou entre pé, sentado e no colo dos pacientes, olhos e ouvidos relaxados, cauda e cabeça erguida, interagindo com os pacientes, tinha interesse em cheirar o ambiente. Ainda assim, o cão 05 descansou em alguns momentos e jogou bola com alguns pacientes.

O cão 01, passava a maior parte do tempo sentado, olhos e ouvidos relaxados, cauda inferior (devido à postura), interagindo com as pupilas assistidas e às vezes estendendo a pata. A postura do cão 04 variou de pé, sentado, deitado e no colo dos alunos, olhos relaxados e às vezes alerta, posição das orelhas oscilava entre relaxado e alerta, cauda levantada e às vezes mais baixa, cabeça erguida, interagia e observava os alunos e se movia quando necessário. O cão 05 balançava entre ficar em pé, sentado, deitado no colo, descontraído os olhos e às vezes alerta, assim como orelhas, cauda e cabeça levantadas, interagiu com os alunos, observou-os de perto, se movia quando necessário e às vezes cheirava o ambiente.

Vale ressaltar que o etograma foi um instrumento inovador e extremamente importante para identificar o comportamento dos terapeutas de cães, através da linguagem corporal, durante as intervenções. Os resultados mostraram que, durante as atividades, os cinco cães analisados não apresentaram alterações comportamentais, tais como: estresse, vocalização, comportamentos passivos, ativos/repetitivos. Perceber que as atividades desenvolvidas não causaram mudanças comportamentais e nem riscos para os assistidos e a equipe envolvida. Semelhante a um estudo desenvolvido por Yamamoto et al. (2012), que avaliou o comportamento de nove cães coterapeutas durante intervenções assistidas por animais, onde tal avaliação não mostrou nenhum comportamento negativo, como estresse ou agressividade.

Mudanças comportamentais podem causar sérios danos à saúde e comprometer o bem-estar animal (Rocha, et al., 2016; Ferreira, 2012). Por isso, é fundamental que o veterinário acompanhe durante as intervenções, para garantir a saúde e o bem-estar dos cães participantes. Por fim podemos afirmar que os cães do projeto pet terapia da UFPel experimentaram o mínimo de estresse, confirmando os achados de Clarck et al. (2020). Além disso observamos que as IAAs para os cães foram estimulantes, pois ao simples preparo dos deles para poderem ir as instituições eles ficavam eufóricos, por isso que observamos o valor maior da frequência 
cardíaca antes das atividades quando comparados no momento pós visita. Resultados semelhantes são descritos por Haubenhofer (2006).

\section{Conclusão}

Conclui-se que intervenções assistidas em animais, desenvolvidas com cães treinados e adaptados aos moradores e ao público assistido, durante o período de 40 a $60 \mathrm{~min}$, com equipe treinada não causam alterações nos sinais vitais e comportamentais dos cães coterapeutas, indicando estresse, garantindo sua saúde e bem-estar animal. Por fim, pode-se ressaltar que em um ambiente com maior espaço interno e uma área externa agradável, onde apenas a equipe de trabalho e atendimento está presente, os cães coterapeutas apresentam uma redução da pressão arterial.

\section{Referências}

Bert, F., Gualano, R. G., Camussi, E., Pieve, G., Voglino, G., \& Siliquini, R. Animal assisted intervention: A systematic review of benefits and risks. European Journal of Integrative Medicine, v.8, ed. 5, October 2016, Pages 695-706, 2016. 10.106/j.eujim.2016.05.005.

Chelini, M. O, \& Otta, E. O. (2016). Terapia assistida por animais. 370 p. Manole.

Clarck, S. D., Smidt, J. M., \& Bauer, B. A. (2019). Welfare considerations: Salivary cortisol concentrations on frequency of therapy dog visits in an outpatient hospital setting: A pilot study. Journal of Veterinary Behavior, 30, 88-91. 10.1016 / j.jveb.2018.12.002

Clark, S. D., Martin, F., Mcgowan, R. G. T. S., Smidt, J. M., Anderson, R., Wang, L., Turpin, T., Langenfeld-Mccoy, N., Bauer, B. A., \& Mohabbat, E. B. (2020). Physiological State of Therapy Dogs during Animal-Assisted Activities in an Outpatient Setting. Animals, 10(5), 819. 10.3390 / ani10050819.

Dotti J. (2014). Terapia e Animais. Noética.

Ferreira J. M. (2012). Cinoterapia na APAE/SG: um estudo orientado pela teoria bioecológica do desenvolvimento humano. Rev. Conhec. y Diver. 4(7). http://dx.doi.org/10.18316/626.

Fries, E., Dettenborn, L., \& Kirschbaum, C. (2009). The cortisol awakening response (CAR): Facts and future directions Int. J. Psychophysiol. 72 (1), 63-73. 10.1016 / j.ijpsycho.2008.03.014

Glenk, L.M., Kothgassner, O. D., Stetina, B.U., Palme, R., Kepplinger, B., \& Baran, H. (2013). Therapy dogs' salivary cortisol levels vary during animal-assisted interventions. Anim. Welf., 22, 369-378. 10.7120 / 09627286.22.3.369.

Haubenhofer, D. K, \& Kirchengast, S. (2006). Excitação fisiológica para cães de companhia que trabalham com seus proprietários em atividades assistidas por animais e terapia assistida por animais. J. Appl. Anim. Welf. Sci. 9, 165-172. 10.1207 / s15327604jaws0902_5.

Kruger, K. A., \& Serpell, J. (2010). Animal-assisted interventions in mental health: definitions and theoretical foundations. Handbook on Animal-Assisted Therapy: Theoretical Foundations and Guidelines for Practice. 3, 33-48. 10.1016/B978-0-12-381453-1.10003-0.

Lima, C. M., Krug, F. D. M., Bender, D. B., Rodrigues, M. R. M., Mechereffe, B. M., Vieira, A. C. G., Capela, S. O., \& Nobre, M. O. (2018). Intervenções assistidas por animais realizadas em ambiente hospitalar na promoção do cuidado com a vida. Revista Expressa Extensão. 23(2), 89-95. https://doi.org/10.15210/EE.V23I2.13189.

Mazzotti, G. A., \& Boere, V. (2009). The right ear but not the left ear temperature is related to stress-induced cortisolaemia in the domestic cat (Felis catus). Laterality, 14, 196-204. https://doi.org/10.1080/13576500802344420.

Nobre, M. O., Krug, F. D. M., Capella, S. O., Ribeiro, V. P., Nogueira, M. T. D., Canielles, C., \& Tilmann, M. T. (2017). Projeto Pet Terapia, Intervenções Assistidas por Animais: uma prática para o benefício da saúde e educação humana. Revista Expressa Extensão, 22(1), 78-89. https://doi.org/10.15210/ee.v22i1.10921.

Pereira G. G. (2015). Etograma de stresse cão e folha de registo, Universidade Lusófona de Humanidades e Tecnologias, Lisboa, Portugual. Dados não publicados.

Reed R, Ferrer L, \& Villegas N. (2012). Curadores Naturais: uma revisão da terapia e atividades assistidas por animais como tratamento complementar de doenças crônicas. Rev. Lat. Amer. de Enfer., 12(3). http://www.scielo.br/pdf/rlae/v20n3/pt_a25v20n3.pdf

Riemer, S., Assis, L., Pike, T. W., \& Mills, D. S. (2016). Dynamic changes in ear temperature in relation to separation distress in dogs. Physiol. Behav. 10.1016 / j.physbeh.2016.09.002

Rocha, C. F. G., Muñoz, P. O. L., \& Roma, R. P. S. (2016). História do relacionamento entre animais humanos e não humanos e da TAA. In: Terapia Assistida por Animais, 370p. 
Research, Society and Development, v. 10, n. 1, e3001019730, 2021

(CC BY 4.0) | ISSN 2525-3409 | DOI: http://dx.doi.org/10.33448/rsd-v10i1.9730

Rosa, P. D. E., Rainho, M. R. G., \& Pereira, G. P. (2015). Revisão sobre Ética e Bem-estar Animal nas Intervenções Assistidas por Cães. Revista Clínica Veterinária. n. 16, ano XX.: https://pesquisa.bvsalud.org/portal/resource/pt/vti-17461

Serpell, J, Coppinger, R., Fine, A. H., \& Peralta, J. M. (2010). Welfare considerations in therapy and assistance animals. Handbook on Animal-Assisted Therapy: Theoretical Foundations and Guidelines for Practice. 3, 481-533. 10.1016 / B978-0-12-381453-1.10023-6

Vasconcelos, A. S. (2016). O bem-estar do animal coterapeuta. In: Terapia Assistida por Animais. 370p.

Yamamoto, K. C. M., Silva, E. Y. T., Costa, K. N., Souza, M. S., Silva, M. L. M., Albuquerque, V. B., Pinheiro, D. M., Bernabé, D. G., \& Olivia, V. N. L. S. (2012). Avaliação fisiológica e comportamental de cães utilizados em terapia assistida por animais (TAA). Arq. Bras. Med. Vet. Zootec. 64(3), 568-576. https://doi.org/10.1590/S0102-09352012000300007 